\title{
Wind Influence on Miro-launcher Dynamics Model
}

\author{
Teodor-Viorel Chelaru ${ }^{l},{ }^{*}$, Valentin Pana ${ }^{I}$, Alexandru Iulian $\underline{\text { Onel }}^{2}$, Tudorel-Petronel \\ Afilipoae $^{2}$, Andrei Filip Cojocaru ${ }^{3}$, and Ionut Cosmin Vasile ${ }^{l}$ \\ ${ }^{1}$ Universiry POLITEHNICA of Bucharest, Faculty of Aerospace Engineering, Romania \\ ${ }^{2}$ National Institute for Aerospace Research "Elie Carafoli" - INCAS, Romania \\ ${ }^{3}$ Deimos Space Romania
}

\begin{abstract}
The paper presents aspects regarding wind influence in dynamics of the three stages micro-launcher. The work is focus on atmospheric turbulence, with dedicated linear model based on characteristics correlation functions, that can be attached to the rigid body model with six degrees of freedom. The results analyzed will be the flight parameters of the launcher, with the wind influence. The novelty of the paper consists in dedicated wind models developed and their implementation in six degrees of freedom micro-launcher model.
\end{abstract}

\section{Introduction}

Since the design stage in the evaluation of the launcher trajectory and in the calculation of the resistance structure must consider the presence of external disruptive factors of the nature of atmospheric turbulence, or some lateral shear currents, which can be overlap or alter with uniform wind areas. One of the characteristics of use of micro-launcher (ML) is that it must be able to be launched in an atmosphere with wind if it does not exceed certain limits. The effects of the large-scale movement (wind) are important in establishing the average flight path. The problem that arises is to determine the trajectory at the gradients of the atmospheric movement (gust). The answer to this is important from three points of view: the determination of a probable trajectory and dispersion around it; the guidance system response and ML structure response to dynamic loads. The problems are: defining wind conditions and turbulence for the description of the velocities field in which the ML flies; defining the aerodynamic forces and moments determined by the velocities field; consider these forces and moments when studying the ML movement or requiring its structure. In the work will be sought to respond to these problems, less to the one related to the request of the structure, customizing the study in the analysis of the wind influence on the ML movement.

\section{DEFINITION OF ATMOSPHERIC TURBULENCE. HYPOTHESES}

Next, we will analyze the influence of atmospheric turbulence on the evolution of ML in the ascending phase. For this we need to define atmospheric turbulence, definition that is based on several simplified assumptions.

The velocity field, variable in time and space, may be considered as result of an average value and a perturbation around it. After the initial phase flight, in the case of long-time flights, the average value may be modelled as referencing the flight with respect to a mobile

\footnotetext{
* Corresponding author: teodor.chelaru@upb.ro
} 
rectangular frame which moves with the wind's average velocity. In a point located by $\bar{r}\left(x_{1}, x_{2}, x_{3}\right)$ with respect to the mobile frame, the air average velocity is:

$$
\bar{u}(\bar{r}, t)=\left[\begin{array}{lll}
u_{1} & u_{2} & u_{3}
\end{array}\right]^{T},
$$

where each component $u_{i}(\bar{r}, t)$ is a four-variable function with random values. It may be described properly by a correlation matrix composed of elements such like:

$$
R_{i, j}(\bar{\xi}, \tau)=<u_{i}(\bar{r}, t) u_{j}(\bar{r}+\bar{\xi}, t+\tau)>
$$

which represents the temporal and spatial average of the product between a component $u_{i}$ located at a point defined by $\bar{r}$ at the time $t$, at the time $u_{j}$ from the point $\bar{r}+\bar{\xi}$ at the time $t+\tau$.

To this matrix we can associate a spectral function matrix whose elements are defined by the Fourier transform function:

$$
\Theta_{i, j}(\bar{\Omega}, \omega)=\frac{1}{(2 \pi)^{4}} \int_{-\infty}^{\infty} \int_{-\infty}^{\infty} \int_{-\infty}^{\infty} \int_{-\infty}^{\infty} R_{i, j}(\bar{\xi}, \tau) e^{-i(\bar{\Omega} \bar{\xi}+\omega \tau)} d \xi_{1} d \xi_{2} d \xi_{3} \mathrm{~d} \tau
$$

The inverse of these elements belongs to the correlation matrix:

$$
R_{i, j}(\bar{\xi}, \tau)=\int_{-\infty}^{\infty} \int_{-\infty}^{\infty} \int_{-\infty}^{\infty} \int_{-\infty}^{\infty} \Theta_{i, j}(\bar{\Omega}, \omega) e^{-i(\bar{\Omega} \bar{\xi}+\omega \tau)} d \Omega_{1} d \Omega_{2} d \Omega_{3} d \omega
$$

Generally, the atmosphere turbulences are not Gaussian. However, many experiments demonstrated that for practical purposes, the normal repartition may be used, producing significant simplifications. On other hand, the statistical parameters $R_{i, j}$ and $\Theta_{i, j}$ of the turbulence are defined in each point in space, $\bar{r}$, and variable with time $t$.

In general, $R_{i, j}$ and $\Theta_{i, j}$ depend on the orientation of the wind-related frame, as for example in the ground boundary layer. If the statistical functions do not depend on space, as in the case of high altitude, the turbulence is considered isotropic. In this situation, the statistical properties in a point are independent of axes orientation, which makes the velocity components square means to be equal:

$$
\sigma^{2}=\overline{u_{1}^{2}}=\overline{u_{2}^{2}}=\overline{u_{3}^{2}}
$$

Where the standard deviation $\sigma$ will be called turbulence intensity.

Because the launch vehicle velocity is much higher than their variation velocity, the ML may fly through an important turbulence zone in such a short time that the turbulent velocities do not change too much, so that the turbulence may be regarded to as ,, a frozen model". This allows us to neglect time in the function $\bar{u}(\bar{r}, t)$, which corresponds to Taylor's hypothesis. Consequently, the correlation and spectral functions become:

$$
R_{i, j}(\bar{\xi}, \tau) \rightarrow R_{i, j}(\bar{\xi}) ; \Theta_{i, j}(\bar{\Omega}, \omega) \rightarrow \Theta_{i, j}(\bar{\Omega}),
$$

and the Fourier integrals formerly defined become from quadruple to triple.

Consequently, the simplest model is the one of homogenous and isotropic turbulences, Gaussian and ,frozen”, a model which is frequently used to analyses the flight outside the ground proximity layer. Inside the ground proximity layer, where ML start occurs, there is necessary to consider anisotropic turbulence.

For the isotropic turbulence, the correlation functions from the correlation matrix may be expressed through Batchelor's relation:

$$
\frac{R_{i j}(\bar{\xi})}{\sigma^{2}}=[f(\xi)-g(\xi)] \frac{\xi_{i} \xi_{j}}{\xi^{2}}+g(\xi) \delta_{i, j},
$$

where $\xi=|\bar{\xi}| ; \delta_{i, j}$-Kroneker's symbol; $\sigma^{2}$ - dispersion, which is the same for each velocity component due to isotropy, while $f$ and $g$ are the characteristic correlation functions.

We denoted, $f(\xi)$ the longitudinal correlation function, given by: 


$$
<u_{1}\left(x_{1}\right) u_{1}\left(x_{1}+\xi_{1}\right) \geq<u_{2}\left(x_{2}\right) u_{2}\left(x_{2}+\xi_{2}\right) \geq<u_{3}\left(x_{3}\right) u_{3}\left(x_{3}+\xi_{3}\right) \geq \cdots
$$

This function represents the correlation between velocity components along an axis, given in two points of that axis (Fig. 1). Based on the hypothesis regarding isotropy, it results that: and:

$$
f_{1}\left(\xi_{1}\right)=f_{2}\left(\xi_{2}\right)=f_{3}\left(\xi_{3}\right)=f(\xi)
$$

$$
f(\xi)=\frac{R_{11}(\xi)}{\sigma^{2}}=\lim \frac{1}{2 X \sigma^{2}} \int_{-X}^{X} u_{1}\left(x_{1}\right) u_{1}\left(x_{1}+\xi\right) d x_{1}
$$

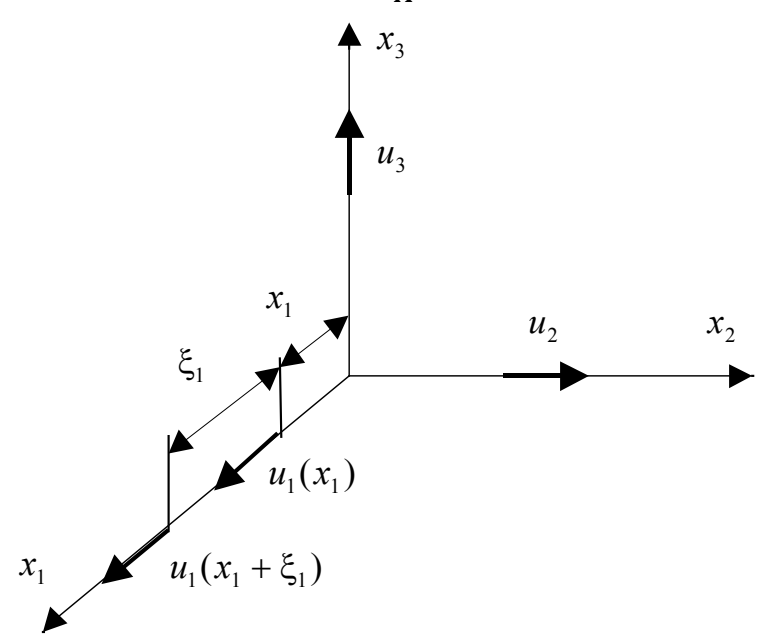

Fig. 1 Longitudinal correlation of velocity components

The other function, $g(\xi)$, is the lateral correlation function given by the relation:

$$
<u_{3}\left(x_{1}\right) u_{3}\left(x_{1}+\xi_{1}\right) \geq<u_{2}\left(x_{1}\right) u_{2}\left(x_{1}+\xi_{1}\right) \geq<u_{3}\left(x_{2}\right) u_{3}\left(x_{2}+\xi_{2}\right) \geq \cdots
$$

and represents the correlation between the transversal velocity components with respect to an axis. Based on the hypothesis regarding isotropy, it results:

that means:

$$
g_{3}\left(\xi_{1}\right)=g_{2}\left(\xi_{1}\right)=g_{3}\left(\xi_{2}\right) \ldots=g(\xi),
$$

$$
g(\xi)=\frac{R_{33}\left(\xi_{1}\right)}{\sigma^{2}}=\lim \frac{1}{2 X \sigma^{2}} \int_{-X}^{X} u_{3}\left(x_{1}\right) u_{3}\left(x_{1}+\xi_{1}\right) d x_{1}
$$

\section{THE UNIFORM TURBULENCE MODEL}

For the characteristic correlation functions, the following relations from work [1] can be used:

$$
f(\xi)=\frac{2^{\frac{2}{3}}}{\Gamma\left(\frac{1}{3}\right)} \zeta^{\frac{1}{3}} K_{\frac{1}{2}}(\zeta) ; \quad g(\xi)=f(\xi)-\frac{2^{-\frac{1}{3}}}{\Gamma\left(\frac{1}{3}\right)} \zeta^{\frac{4}{3}} K_{\frac{2}{3}}(\zeta)
$$


where: $\zeta=\frac{\xi}{a L}$, with $a=1,339$ and $L$ representing the characteristic length. $\Gamma$ is the seconddegree Euler function and $K_{v}$ is the second degree Bessel modified function. The characteristic functions defined by (14) are graphically represented in Fig. 2.

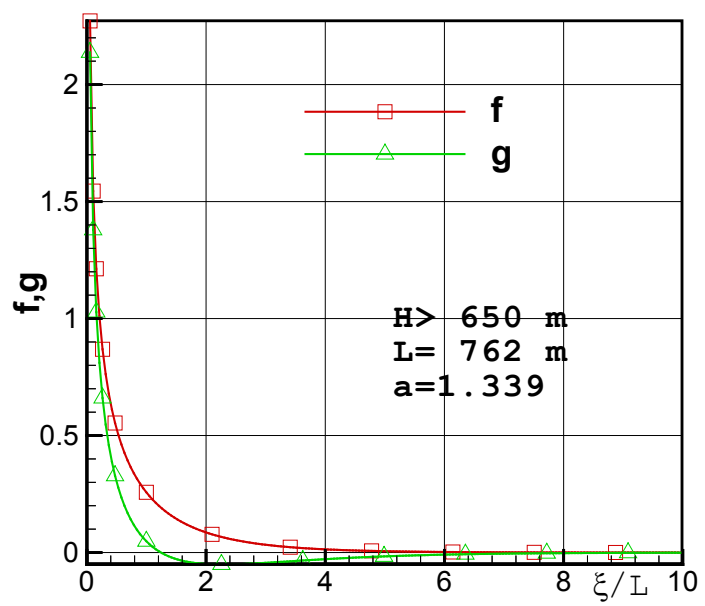

Fig. 2 Characteristics correlation functions

The Fourier transforms of the functions $f(\xi)$ and $g(\xi)$ are spectral one-dimensional functions related to longitudinal and lateral coordinates:

$$
\Phi_{11}\left(\Omega_{1}\right)=\frac{\sigma^{2}}{\pi} \int_{0}^{\infty} f(\xi) e^{-i \Omega_{1} \xi} d \xi ; \quad \Phi_{33}\left(\Omega_{1}\right)=\frac{\sigma^{2}}{\pi} \int_{0}^{\infty} g(\xi) e^{-i \Omega_{1} \xi} d \xi
$$

These functions are presented in Fig. 3

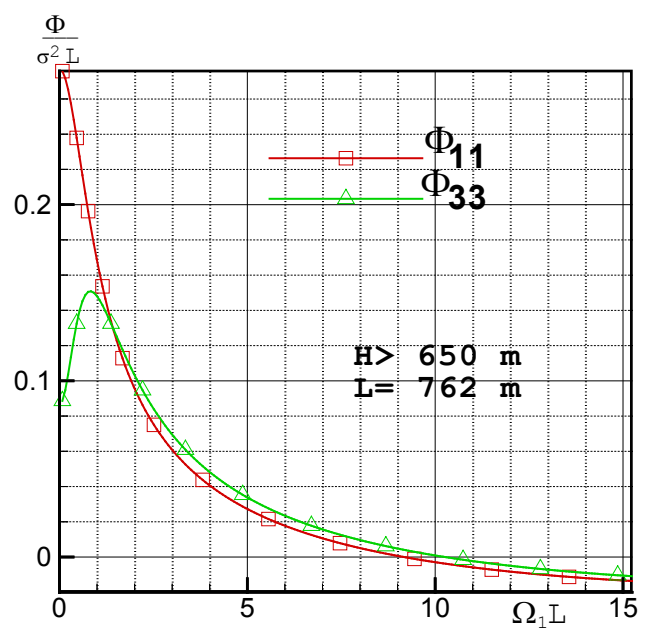

Fig. 3 Characteristic spectral functions

For the adopted models, both theoretical or experimental, we defined the characteristic length $L$ and the turbulence intensity $\sigma$. In case of altitudes over $650 \mathrm{~m}$ the boundary layer influence is diminished, and the atmosphere may be considered, a unique value for the characteristic length $L$ being recommended, both to longitudinal and lateral spectral functions. For the model considered, according to [1] $L=762 \mathrm{~m}$. The standard deviation $\sigma$ is the same along all the three directions, depending only on the turbulence intensity: - low turbulence; - mean turbulence; - height turbulence (storm). 
The turbulence model obtained in this way is the simplest possible, being known as the uniform field model, in which only the linear components of velocity along the three axes, reduced in the gravity center of the ML, are considered. Each component is considered uniform in each point of ML's surface.

\section{THE LINEAR TURBULENCE MODEL}

A higher order approximation is the one of a linear field, the turbulence velocities being considered as linear functions depending on position.

Authors of [1] and [2] developed in case of airplane the linear turbulence models in four points. These models also allow calculation of rotation velocity components $p_{g}, q_{g}, r_{g}$ due to turbulence.

Starting from the method proposed in [2], in work [3] is developed for rockets a linear model of turbulence in two points which allow deducing of angular velocity of pitch and yaw motion. We briefly present this model in what follows.

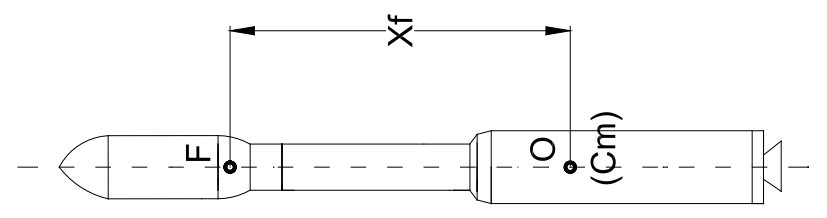

Fig. 4 Two-point ML model

In the linear turbulence case, due to the slender axis-symmetrical shape of the launcher, some gradients may be neglected. The influence of the remaining gradients may be considered as like supplementary rotation velocities in pitch and yaw. To calculate these velocities, we shall consider a model of the ML in two points as shown in Fig. 4. where point 0 represents the position of the mass center and $F$ represents the position of the configuration focal point.

The length $l_{v} \equiv X_{f}$, called aerodynamic length, represents the distance from the mass center to the focal point. Based on this model, the pitch and yaw velocities, equivalent to the velocity gradients, are:

$$
r_{g}=\frac{1}{l_{v}}\left(v_{0}-v_{1}\right), q_{g}=\frac{1}{l_{v}}\left(w_{1}-w_{0}\right)
$$

to which there are added the linear velocities due translation for the uniform turbulence:

$$
w_{g}=w_{0} ; u_{g}=u_{0} ; v_{g}=v_{0}
$$

To calculate the velocities produced by turbulence, we expressed their correlation functions through the characteristic functions $f$ and $g$, by doing as in the case of uniform distribution, we calculated the spectral functions corresponding to the correlation functions and, from these, we found some possible forms of the translation and rotation velocities generated by turbulence.

For the case of the yaw turbulence velocity the following initial relation was used:

$$
R_{q q}(\tau)=<q_{g}(t) q_{g}(t+\tau)>
$$

obtaining:

$$
\frac{\left(l_{v}\right)^{2}}{\sigma^{2}} R_{q q}(\tau)=2 g\left(\xi_{1}\right)-g\left(\xi_{2}\right)-g\left(\xi_{3}\right)
$$

where:

$$
\xi_{1}=V \tau \xi_{1}=V \tau ; \quad \xi_{2}=\xi_{1}+l_{v} ; \xi_{3}=\xi_{1}-l_{v} .
$$

To calculate the angular pitch velocity, a similar procedure was used: 


$$
\begin{aligned}
R_{r r}(\tau)=\frac{1}{\left(l_{v}\right)^{2}}< & \left(v_{0}-v_{1}\right)\left(v_{0}^{\prime}-v_{1}^{\prime}\right)>=\frac{1}{\left(l_{v}\right)^{2}}\left[<v_{0} v_{0}^{\prime}>+<v_{1} v_{1}^{\prime}>-<v_{1} v_{0}^{\prime}>-\right. \\
& \left.<v_{0} v_{1}^{\prime}>\right]
\end{aligned}
$$

from which we obtained:

$$
\frac{\left(l_{v}\right)^{2}}{\sigma^{2}} R_{r r}(\tau)=2 g\left(\xi_{1}\right)-g\left(\xi_{2}\right)-g\left(\xi_{3}\right),
$$

From the previous relations and (14) for the characteristic functions $f$ and $g$, the correlation functions for the rotation velocities were determined. The two rotation velocity components are equal, $R_{q q}=R_{r r}$, due to axial symmetry and can be represented by a single correlation function in Fig. 5.

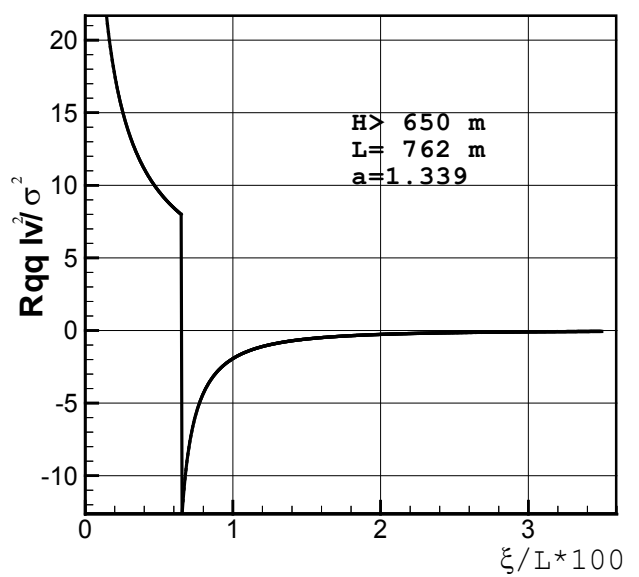

Fig. 5 Correlation function for rotation velocities

Starting from the correlation function determined as above, by Fourier Transform we obtained the spectral function for the rotation velocity, represented in Fig. 6

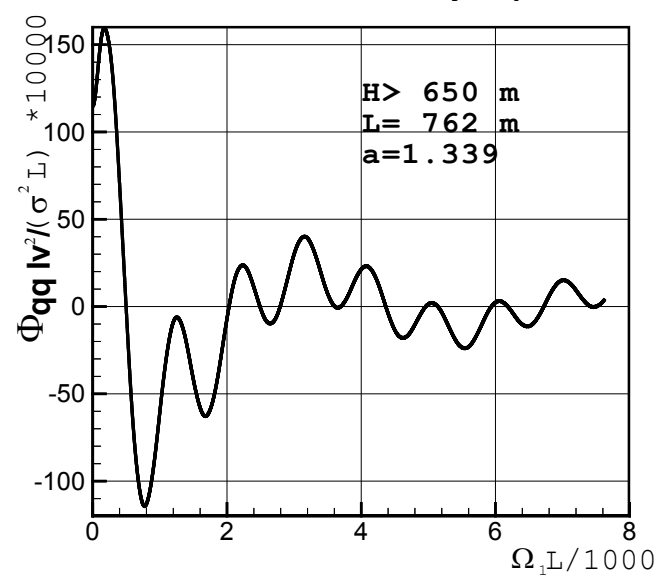

Fig. 6 Spectral function for the rotation velocity

For check-up our calculus method, we compare the velocity components obtained with the linear turbulence field theory to those obtained using the uniform field theory. These proved to be identical. Unlike these, the relationships found for angle velocities (19),(20) can be used only we work with the theoretical model defined by the characteristic functions (14). In the case of symmetrical axial configurations, the only one that appears is the term due to the longitudinal velocity angle of pitch/yaw (19),(20), and if the aerodynamic length is small 
relative to the characteristic length, its influence it is small and to use the linear model not justified. In the case of large-length rockets this term can become important, and for airplane configurations, as shown in [1] and [2] other coupling terms may appear, the use of the linear model being indicated.

\section{GENERATING ATMOSPHERIC TURBULENCE}

To generate atmospheric turbulence, we will use reverse Fourier transform in so call method of sinusoidal sum [3]. The basic idea of this method is that starting from the spectral function module, the amplitude of oscillation can be obtained for a certain frequency band, and thus generate one of the possible forms of that size, the shape to which we still need the initial phase. If we start from the modules of spectral functions presented in Fig. 3, by integrating the portions we will determine the value of the amplitude corresponding to a frequency band, which can be approximated with the corresponding frequency of integrated area center. In addition, if we choose the integral of equal size, the values of the amplitudes obtained will be the same, differing only the frequency value of the band. To customize this model, each frequency obtained will be associated with an initial phase, which is arbitrarily chosen. Thus, if we note with $S_{\varphi}$ portion of the area obtained by integrating the module of a spectral function from Fig. 3, and we will take into account that the function was represented only for positive values of the argument $\Omega_{1}$, we can write:

$$
A=\sigma \sqrt{2 S_{\varphi}}
$$

two of the translation velocities generated by turbulence at a point $\xi$ being given by:

$$
u_{g}(\xi)=A_{1} \sum_{i} \sin \left(\Omega_{1}^{i} \xi+\varphi_{1}^{i}\right) ; \quad w_{g}(\xi)=A_{2} \sum_{i} \sin \left(\Omega_{2}^{i} \xi+\varphi_{2}^{i}\right)
$$

where the index "1" attributed to the amplitude, pulsations and initial phases obtained from the longitudinal spectral function module, and the index " 2 " elements obtained from the lateral spectral function module. The third component of the speed, except for the initial phase coincides with the second one, being generated by the same spectral function:

$$
v_{g}(\xi)=A_{2} \sum_{i} \sin \left(\Omega_{2}^{i} \xi+\varphi_{3}^{i}\right)
$$

If the launcher is moving straight, with the velocity $V$, the linear size can be expressed depending on time by the relationship:

$$
\xi=V \tau
$$

Turbulence velocities can thus be expressed as time functions. In Fig. 7 the axial velocity $u_{g}$ and the normal velocity $w_{g}$ generated by turbulence are presented.

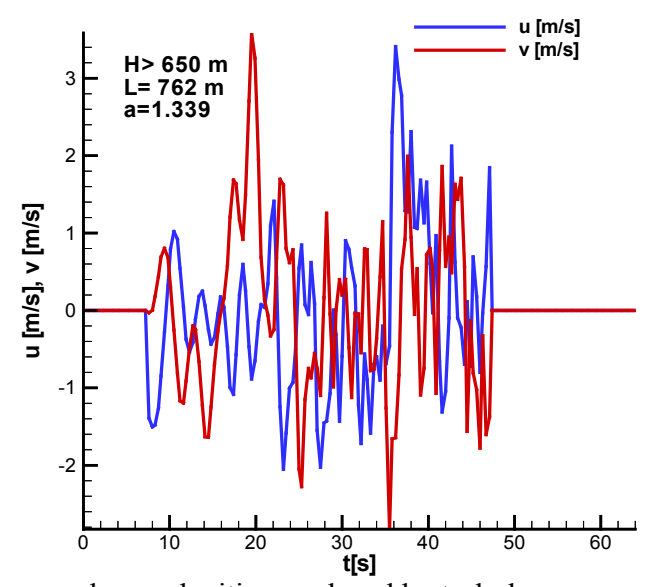

Fig. 7 The axial $u_{g}$ and normal $w_{g}$ velocities produced by turbulence 
By applying similar relationships to the (22),(23) one of the possible developments in time of the components of the rotation velocity generated by turbulence is obtained. Since for rotation velocities the spectral functions are equal, as evidenced by the Fig. 6, the velocity in yaw will have a similar shape to the pitching, $r_{g} \approx q_{g}$, differences only through the initial phases.

\section{CONCLUSIONS}

In the work we developed two models of turbulence, one uniform, in which only the translation velocity of the center of the mass are taken into consideration and a linear one, in which in addition to the translation velocity, longer appear terms due to the longitudinal angle velocities (pitch, yaw) around mass center.

If the aerodynamic length is small in relation to the characteristic length, its influence is secondary, and to the use of the linear model not justified.

In the case of large-length missiles, as in ML case, this term can become important, and for airplane configurations. The turbulences model obtained can be added to dynamic flight ML model 6 DOF

As shown in [1] and [2], for airplane case, other coupling terms may appear, the use of the linear model is necessary.

In the work [3], in addition to developments related to trajectory problems in turbulence conditions, using the previously presented model, dynamic test specifications have been developed to check the ground missile resistance structure, stage important in designing a rocket in that category.

\section{References}

[1] B. ETKIN, Dinamics of Atmospheric Flight, New York: John Wiley \& Sons, Inc., (1972).

[2] T.V. CHELARU, Dinamica Zborului - Proiectarea avionului fără pilot, Bucureşti: Ed. Printech, (2003), p. 308.

[3] M. Cernat M., T.V. Chelaru, "Turbulent Atmosphere Influence on Anti-Hailstone Rocket Flight," Journal of Battlefield Technology, vol. 11, no. 1, pp. 1-8, March (2008). 